\title{
Teoría de la Conversación Literaria de Aidan Chambers como Estrategia para el Fortalecimiento de la Comprensión Lectora
}

\author{
Aidan Chambers Theory of Literary Conversation as a Strategy for Strengthening \\ Reading Comprehension
}

Omaira Peña-Maldonado

\section{- EDICIÓN. Pesiliencia}

Recibido: 24/agosto/2021

Aceptado: 24/noviembre/2021

Publicado: 29/noviembre/2021

Páginas: 183-190

4aís

${ }^{1}$ Colombia

\section{IIIII Institución \\ ${ }^{1}$ Universidad de Pamplona}

\section{Correo Eletrónico}

1omix08p@gmail.com

\section{ORCID}

'https://orcid.org/0000-0001-9372-5139

\section{Citar así: Lf APA / IEEE}

Peña-Maldonado, O. (2021). Teoría de la Conversación Literaria de Aidan Chambers como Estrategia para el Fortalecimiento de la Comprensión Lectora. Revista Tecnológica-Educativa Docentes 2.0, 1(1), 183-190.

https://doi.org/10.37843/rted.v1i1.273

O. Peña-Maldonado, "Teoría de la Conversación Literaria de Aidan Chambers como Estrategia para el Fortalecimiento de la Comprensión Lectora", RTED, vol. $1, \mathrm{n}$. 1, pp. 183-190, nov. 2021.

\section{Resumen}

La escasa comprensión lectora es una de las principales problemáticas que afrontan los docentes de primaria. Además, es uno de los factores limitantes en el desempeño académico de los niños en Colombia. Con esta investigación se analizó la incidencia de la teoría de la conversación literaria de Aidan Chambers en el fortalecimiento de la comprensión lectora en el grado tercero, Sede R Jagüi del Colegio Juan XXIII. La metodología implementada se basó en el paradigma metodológico cualitativo, Investigación Acción (IA), sustentado en un análisis crítico con la participación activa de los agentes implicados. La investigación fue realizada con un grupo de dos discentes. Se ejecutó a través de la modalidad de enseñanza totalmente virtual utilizando la plataforma de Zoom, WhatsApp y Educaplay, por medio de videos, libros álbum, composiciones, juegos, creaciones. El estudio fue realizado con una muestra de 2 estudiantes del grado tercero, sus edades oscilan entre los 8 y 9 años. Se planteó en la unidad didáctica de cinco sesiones de trabajo en pro de mejorar la comprensión lectora y abordar una serie de temáticas sobre el territorio, a partir de las cuales elaboraron sus propias producciones para alimentar la página el producto final (una página web). La propuesta fue efectiva, así lo dejan ver los resultados de la prueba aplicada en la cual se puede evidenciar las estrategias innovadoras que permitieron mejorar la comprensión de los hábitos lectores.

Palabras clave: Conversación literaria, Aidan Chambers, estrategias, comprensión lectora.

\section{Abstract}

Poor reading comprehension is one of the main problems faced by primary school teachers. In addition, it is one of the limiting factors in the academic performance of children in Colombia. This research analyzed the incidence of Aidan Chambers' theory of literary conversation in strengthening reading comprehension in the third grade, Sede R Jagüi of Colegio Juan XXIII. The implemented methodology was based on the qualitative methodological paradigm, Action Research (IA), supported by a critical analysis with the active participation of the agents involved. The research was carried out with a group of two students. It was executed through the virtual teaching modality using the Zoom, WhatsApp, and Educaplay platform, through videos, album books, compositions, games, creations. The study was carried out with a sample of 2 third-grade students; their ages range between 8 and 9 years. It was proposed in the didactic unit of five work sessions to improve reading comprehension and address a series of themes about the territory. They made their productions to feed the final product (a web page) on the page. The proposal was effective, as can be seen from the results of the applied test in which it is possible to show the innovative strategies that allowed to improve the understanding of reading habits.

Keywords: Literary conversation, Aidan Chambers, strategies, reading comprehension. 


\section{Introducción}

La escasa comprensión lectora es una de las principales problemáticas que afrontan los docentes de primaria. Además, es uno de los factores que limitan el desempeño académico de los niños en Colombia. Se observa con frecuencia el bajo nivel de comprensión lectora, notándose en éstos la dificultad para interpretar o inferir un texto. Este proyecto de investigación se ocupa de esta problemática en la Institución Educativa Colegio Juan XXIII del Municipio de Macaravita Santander sede R Jagüi, en el grado tercero. Las estadísticas del área de lenguaje en los tres componentes pragmáticos, semántico y sintáctico, aplicadas por Icfes (2018) en las pruebas Saber $3^{\circ}, 5^{\circ}$ y $9^{\circ}$ dicen que un $73 \%$ de los estudiantes muestran resultados bajos en el proceso de lectura, así mismo a nivel departamental un $45 \%$ presentan bajo rendimiento en esta área.

La baja comprensión lectora en los estudiantes del grado tercero se da por la falta de practica en la lectura: Salazar \& Ponce (1999) expresan, cuando el niño lee poco, tienen una limitada practica en el hábito de leer, pero la poca lectura no solo es problema de los niños, los adultos tampoco leen como se espera. Arias (2021) habla de los retos de la educación en Colombia, considerando la educación rural como forma de consumo de tecnología, como la posibilidad de adquirir técnicas de ingreso a la sociedad letrada, una manera de construir los procesos de aprendizaje de la vida cotidiana, lejos de las prácticas sociales de sus habitantes, quienes asumen tecnologías que no suelen ser familiares con la ruralidad, transmiten un ideario de cercanía al mundo modernizado, en el cual la educación rural transmite un currículo general, de carácter nacional, que deja por fuera el saber propio de sus pobladores.

Esta propuesta investigativa, parte de la oralidad, el gusto por la lectura de forma cooperativa con la intención de crear ambientes literarios. Chambers (2017) afirma no estar interesado en la conversación en sí, sino en el papel que juega en la vida de los lectores. Discriminan, piensan, gozan, cree que la lectura como un todo es una actividad mucho más productiva, mucho más valiosa. Pero también la conversación es esencial en nuestras vidas, en buena medida la mayoría de nosotros, como suele decirse, no sabemos pensar hasta oírnos diciéndolo. Hablar bien sobre los libros es una actividad en sí misma muy valiosa, pero también es el mejor entrenamiento para conversar sobre otras cosas. Al ayudar a los niños a hablar de sus lecturas, los ayudamos a expresarse acerca de todo lo otro que hay en sus vidas.

Con esta investigación se analizó la incidencia de la teoría de la conversación literaria de Aidan Chambers en el fortalecimiento de la comprensión lectora en el grado tercero, Sede R Jagüi del Colegio Juan XXIII. Adquirir el hábito por la lectura, comprender lo leído, con un proceso más dinámico e innovador, conlleva a crear un lector competente, que interprete, impugne, plantee, así como la mejoría en los resultados en las diferentes pruebas aplicadas por el Ministerio de Educación, a su vez mejorar la calidad de en los diferentes niveles de escolaridad. La comprensión lectora es un elemento fundamental para el éxito académico de los alumnos de primaria, impacta sus oportunidades educativas, de trabajo, de inserción social a lo largo de su vida.

\section{Metodología}

La metodología implementada en esta investigación se basó en el paradigma metodológico cualitativo. Por lo que, los autores Strauss, \& Corbin (2002), señalan que un paradigma no es más que "una perspectiva que se adopta sobre los datos, otra posición analítica que ayuda a recolectarlos y ordenarlos de manera sistemática" (2002, p.140). Escudero \& Cortez (2018) afirman que la investigación cualitativa se caracteriza por ser interpretativa, se lleva a cabo en determinados grupos sociales, con una participación activa durante todo el desarrollo del proceso investigativo, además es flexible en relación con que se ajusta a los sucesos para lograr una correcta interpretación de datos 
descriptivos, su metodología se fundamenta en la recolección de información no numérica, basada en descripciones, observaciones, entrevistas e imágenes.

Según Herrera (2010), la investigación cualitativa podría entenderse como una categoría de diseños de investigación que extrae descripciones a partir de observaciones que adoptan la forma de entrevistas, narraciones, notas de campo, grabaciones, transcripciones de audio y video casetes, registros escritos de todo tipo, fotografías o películas y artefactos. Se utilizan los siguientes métodos: fenomenología, etnografía, teoría fundamentada, etnometodología investigación-acción y método biográfico. Además, Durango-Herazo señala que la investigación cualitativa "llevada a cabo por el docente en la escuela lo orienta a renovar constantemente su praxis pedagógica" (2015, p.44).

El tipo de investigación fue la Investigación - Acción (IA). Su propósito fundamental se centra en aportar información y guía la toma de decisiones para programas, procesos y reformas estructurales. Sandín (2010) señala que la investigación acción pretende, esencialmente, propiciar el cambio social, transformar la realidad y que las personas tomen conciencia de su papel en ese proceso de transformación. Para Montero \& León (2002) representa el estudio de un contexto social donde mediante un proceso de investigación con pasos "en espiral", se investiga al mismo tiempo que se interviene. Lo expuesto por el autor Salazar (1992), señala que IA permite a los investigadores basar sus observaciones en la convivencia con las comunidades, así como el adquirir sus conocimientos en el transcurso de la investigación en su propio territorio a través del aprendizaje significativo.

La investigación fue realizada con una muestra de dos estudiantes del grado tercero, en la institución Educativa Sede R Jagüi del Colegio Juan XXIII del municipio de Macaravita Santander. Sus edades oscilan entre los 8 y 9 años. Se utilizó el muestreo no probabilístico, caracterizado por determinar muestras no representativas que se determinan o calculan de forma arbitraria
(Pimienta-Lastra, 2000). No se puede utilizar el método al azar ya que la muestra de la investigación se obtuvo a través de la formula estadística población finita. La Investigación se desarrolló a partir de las siguientes fases:

Fase 1: Observar. Se estructuró desde la construcción de un bosquejo del problema y la recolección de datos. Esta fase dio cumplimiento al primer objetivo específico de esta investigación identificando las necesidades, oportunidades, niveles de lectura de los estudiantes desde la observación directa, prueba diagnóstica, entrevista de conocimiento. Se aplicó para conocer el nivel y gusto por la lectura.

Fase 2: Pensar. Esta fase permitió analizar e interpretar y formular un plan o programa para resolver el problema, dando cumplimiento al objetivo específico dos donde se plantea el diseño de una unidad didáctica y permite la consolidación de una estrategia para fortalecer la comprensión lectora de los estudiantes del grado tercero a partir de la incidencia de la teoría de Aidan Chambers.

Fase 3: Actuar. Esta fase permitió resolver problemas e implementar mejoras, ejecutar el plan o programa y evaluar los resultados, dando cumplimiento al objetivo específico tres planteado en esta investigación, llevando a cabo la ejecución de la unidad didáctica así como la evaluación de esta en los estudiantes del grado tercero de la sede R Jagüi colegio Juan XXIII del de Municipio de Macaravita Santander.

Fase 4: Reflexionar. Conduce a la evaluación y conclusiones. Estas fases se dieron de manera cíclica, una y otra vez, hasta que el problema fue resuelto, el cambio se logró satisfactoriamente. Finalmente la reflexión sobre la ejecución de la unidad didáctica permitió la evaluación a partir de la ejecución que conllevo, además, establecer las conclusiones y recomendaciones con base en los resultados obtenidos.

Ciertamente, el desarrollo de estas cuatro fases permitió la consolidación de una estrategia para el fortalecimiento de la comprensión lectora en los estudiantes del grado tercero de la sede R Jagüi Colegio juan XXIII y las prácticas educativas que allí se 
adelantan. La investigación se ejecutó a través de la modalidad de enseñanza totalmente virtual utilizando la plataforma de Zoom, WhatsApp y Educaplay, por medio de videos, libros álbum, composiciones, juegos, creaciones. La unidad didáctica, con cinco sesiones de trabajo, permitió a los estudiantes mejorar la comprensión lectora y abordar una serie de temáticas sobre el territorio, a partir de las cuales realizaron sus propias producciones.

\section{Resultados}

Con el propósito de demostrar el impacto de la propuesta de investigación fue necesario realizar un seguimiento a los diferentes momentos, los cuales fueron analizados tomando como referencia la prueba diagnóstica y la encuesta de conocimiento (Tabla 1, Figura1, Figura 2 y Figura 3), a través de cada una de las actividades propuestas en la unidad didáctica conformada por cinco sesiones, desarrolladas y analizadas a su vez, a partir de tres momentos a saber:

La fase de exploración permitió partir de los presaberes de los estudiantes así como la introducción al tema a tratar durante la secuencia a desarrollar. Esta fase aportó actividades optimando la comprensión lectora desde la fundamentación teórica, la lectura de textos, la conversación en torno a la categoría que se estaba desarrollando. La fase de cierre evidenció los elementos adquiridos con el desarrollo de la secuencia, la evaluación de los trabajos finales elaborados por los estudiantes que sirvieron de fundamento para mejorar la comprensión lectora.

El análisis detallado de esta información dio paso a identificar las fortalezas, debilidades evidenciadas en el desempeño de los estudiantes con el propósito de mejorar la comprensión lectora desde las categorías propuestas. Este análisis permitió verificar los resultados, realizar la interpretación para identificar, valorar el nivel de impacto de la propuesta de investigación al final del proceso. Tal como lo sustentas los autores Moreno-Muñoz \& ValverdeCaravaca, "la modalidad de literatura como son los cuentos despiertan en la población el deseo de leer" (2004, p.1).

Los resultados se presentaron durante la etapa final de esta investigación mediante un análisis cualitativo y la modalidad de Investigación Acción, corresponde ahora realizar el análisis de la información recolectada durante la implementación de la unidad didáctica, y presentar los resultados, con la intención de responder la pregunta planteada. Este informe se centró en precisar en qué medida la teoría de Aidan Chambers permitió mejorar las prácticas de comprensión lectora en los estudiantes del grado tercero de la sede R Jagüi del Colegio Juan XXIII. Toda esta información constituyo el referente sobre el cual se formularon las conclusiones y recomendaciones del trabajo investigativo.

La prueba diagnóstica busca identificar las necesidades, oportunidades, niveles de lectura de los estudiantes desde una perspectiva de reconstrucción para fortalecer la comprensión lectora. A continuación se presentan los resultados del instrumento aplicado a dos estudiantes del grado tercero de la sede R Jagüi del Colegio Juan XXIII, consiste en la observación de un audio video titulado La batalla del grillo y el oso, en base a ello la solución de interrogantes planteados. A continuación se presenta el análisis detallado de cada una de las interrogantes planteadas en la Tabla 1.

Una vez analizada la prueba realizada por los estudiantes se puede deducir: un $35 \%$ de las respuestas fueron correctas mientras que un $65 \%$ de las mismas fueron incorrectas. Se afirma el planteamiento del objetivo específico uno para llegar a cumplir al objetivo general planteado, estructurando actividades didácticas que permitan mejorar la comprensión lectora en los estudiantes del grado tercero de la sede $\mathrm{R}$ Jagüi del colegio Juan XXIII.

Para este apartado el análisis de la información recolectada se realizó a través del aplicativo de docs.google.com aplicando este instrumento de encuesta a los dos estudiantes del grado tercero de la sede R Jagüi cuyos resultados se aprecian en las figuras 1, Figura 2 y Figura 3. 


\section{Tabla 1}

\section{Prueba Diagnóstica}

\begin{tabular}{|c|c|c|c|c|c|}
\hline & frecuencia & & & porcentaje & \\
\hline Preguntas analizadas & correctas & incorrectas & $\begin{array}{l}\text { Total } \\
\text { frecuencia }\end{array}$ & correctas & incorrectas \\
\hline $\begin{array}{l}\text { ¿Después de haber visto el video } \\
\text { cuento la batalla del grillo y el oso } \\
\text { se puede inferir y el oso es un } \\
\text { personaje? }\end{array}$ & 1 & 1 & 2 & $50 \%$ & $50 \%$ \\
\hline $\begin{array}{l}\text { ¿Dónde estaba el grillo cuando fue } \\
\text { golpeado? }\end{array}$ & 0 & 2 & 2 & $0 \%$ & $100 \%$ \\
\hline ¿A quién desafío el grillo? & 1 & 1 & 2 & $50 \%$ & $50 \%$ \\
\hline ¿Cuántas cajas abrió el grillo? & 0 & 2 & 2 & $0 \%$ & $100 \%$ \\
\hline $\begin{array}{l}\text { ¿Para enfrentar el oso el grillo } \\
\text { decidió? }\end{array}$ & 1 & 1 & 2 & $50 \%$ & $50 \%$ \\
\hline $\begin{array}{l}\text { ¿La fábula la batalla del grillo y el } \\
\text { oso es una adaptación de? }\end{array}$ & 0 & 2 & 2 & $0 \%$ & $100 \%$ \\
\hline ¿La historia es desarrollada? & 1 & 1 & 2 & $50 \%$ & $50 \%$ \\
\hline ¿Los personajes principales son? & 1 & 1 & 2 & $50 \%$ & $50 \%$ \\
\hline ¿Por qué perdió el oso la disputa? & 0 & 2 & 2 & $0 \%$ & $100 \%$ \\
\hline $\begin{array}{l}\text { ¿La fábula son historias y siempre } \\
\text { le dejan a la lectora una? }\end{array}$ & 2 & 0 & 2 & $100 \%$ & $0 \%$ \\
\hline Total preguntas analizadas & 7 & 13 & 20 & $35 \%$ & $65 \%$ \\
\hline
\end{tabular}

Nota. Analizas de los resultados a los interrogantes planteados durante la prueba diagnóstica, elaboración propia (2021).

\section{Figura 1}
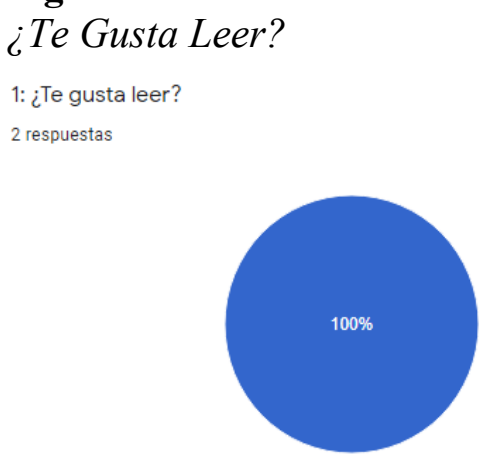

Nota. Primera pregunta de la encuesta realizada a los estudiantes del grado tercero: Lo anterior confirma que los estudiantes leen con frecuencia algún tipo de texto o información, lo cual fortalece el desarrollo investigativo, elaboración propia (2021).

\section{Figura 2}

\section{¿Qué Libros Prefieres Leer?}

\section{2: ¿Qué libros prefieres leer?}

2 respuestas

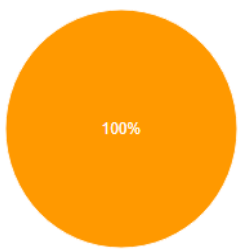

- Libros de aventura - Libros de novelas Libros de cuentos

\section{Figura 3}

\section{¿Cómo Calificarías tu Interés por la Lectura?}

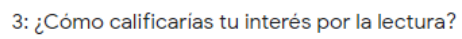

3: ¿Cómo calificarias tu interés por la lectura?

2 respuestas

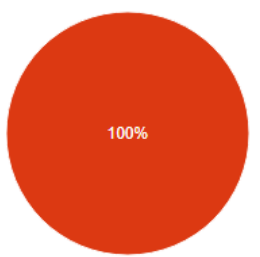

- Alto

Medio Bajo

Nota. En lo que respecta a calificar el interés por la lectura el $100 \%$ de los estudiantes encuestados manifestaron un nivel medio, en este sentido es importante mencionar que es deber del docente implementar estrategias y motivar al estudiante a mejorar su capacidad lectora, elaboración propia (2021).

La fase de planeación permitió diseñar una unidad didáctica y consolidar una estrategia para fortalecer la comprensión lectora de los estudiantes del grado tercero a partir de la incidencia de la teoría de Aidan

Nota. Los encuestados prefirieron los libros de cuentos, afirmación que favorece la presente investigación, elaboración propi (2021). 
Chambers con el cual se pudo cumplir lo planteado. Sustentado por lo expuesto por Reyes "la lengua es la fuente de todos los misterios en la infancia y cuando un niño la apropia, es uno de los trabajos más complejos y apasionantes y es por eso, que la poesía... son el primer acopio" (2017, p.1).

La fase 0 , presentación formal de la investigación, fue el momento en el que se facilitó a la rectora de la institución, padres de familia y estudiantes. Así mismo, los padres de familia firmaron el consentimiento informado, autorizando a los niños para participar de las actividades programadas, explicando que toda la información sería utilizada solo para los fines educativos de la propuesta planteada con el propósito de presentar un intercambio de mensajes orales (Silva De Marco, 2004).

La fase 1 denominada previa $\mathrm{o}$ diagnostica se aplicó la prueba de verificación del nivel de comprensión lectora en los estudiantes, al igual que la encuesta de conocimiento. Seguidamente la fase dos planeaciones, bajo el título "memorias de mi territorio".

Para tal efecto, se aplicó una prueba de comprensión lectora con el ánimo de verificar los resultados (ver Tabla 2) después de haber desarrollado las actividades de mejora propuestas, ya que lo expuesto por el autor Puerto "el texto literario es una herramienta que ofrece elementos para pensarse y reflexionar sobre el mundo" (2015, p.1).

Tabla 2

Prueba de Comprensión Lectora

\begin{tabular}{|c|c|c|c|c|c|}
\hline & Frecuencia & & & Porcentaje & \\
\hline Preguntas analizadas & Correctas & Incorrectas & $\begin{array}{l}\text { Total } \\
\text { frecuencia }\end{array}$ & Correctas & Incorrectas \\
\hline $\begin{array}{l}\text { ¿En la batalla del grillo y el oso se } \\
\text { mencionan los valores de? }\end{array}$ & 2 & 0 & 2 & $100 \%$ & $0 \%$ \\
\hline $\begin{array}{l}\text { ¿Cuándo el grillo estaba tomando el } \\
\text { sol en el camino fue golpeado por? }\end{array}$ & 2 & 0 & 2 & $100 \%$ & $0 \%$ \\
\hline $\begin{array}{l}\text { ¿Los animales que muestran las } \\
\text { imágenes ayudan a? }\end{array}$ & 2 & 0 & 2 & $100 \%$ & $0 \%$ \\
\hline $\begin{array}{l}\text { ¿De la caja que abrió el grillo, } \\
\text { salieron? }\end{array}$ & 1 & 1 & 2 & $50 \%$ & $50 \%$ \\
\hline $\begin{array}{l}\text { ¿Para enfrentar al oso el grillo } \\
\text { decidió? }\end{array}$ & 2 & 0 & 2 & $100 \%$ & $0 \%$ \\
\hline $\begin{array}{l}\text { ¿La fábula la batalla del grillo y el } \\
\text { oso es una adaptación de? }\end{array}$ & 1 & 1 & 2 & $50 \%$ & $50 \%$ \\
\hline ¿La historia es desarrollada? & 2 & 0 & 2 & $100 \%$ & $0 \%$ \\
\hline ¿Los personajes de la fábula son? & 2 & 0 & 2 & $100 \%$ & $0 \%$ \\
\hline ¿Por qué perdió el oso la disputa? & 1 & 1 & 2 & $50 \%$ & $50 \%$ \\
\hline $\begin{array}{l}\text { ¿El orden en que sucedió la historia } \\
\text { es? }\end{array}$ & 2 & 0 & 2 & $100 \%$ & $0 \%$ \\
\hline $\begin{array}{l}\text { ¿Cuándo la historia deja una } \\
\text { enseñanza o moraleja hablamos de? }\end{array}$ & 1 & 1 & 2 & $50 \%$ & $50 \%$ \\
\hline $\begin{array}{l}\text { ¿De acuerdo con las siguientes } \\
\text { imágenes se afirma que se trata de? }\end{array}$ & 1 & 1 & 2 & $50 \%$ & $50 \%$ \\
\hline $\begin{array}{l}\text { Es una narración breve oral o escrita, } \\
\text { en la que se narra una historia de } \\
\text { ficción con un reducido número de } \\
\text { personajes, una intriga poco } \\
\text { desarrollada y un clímax que } \\
\text { desenlace finales rápidos. ¿Por lo } \\
\text { anterior nos invita a pensar que es? }\end{array}$ & 0 & 2 & 2 & $0 \%$ & $100 \%$ \\
\hline Total preguntas analizadas & 19 & 7 & 2 & $73 \%$ & $27 \%$ \\
\hline
\end{tabular}

Nota. Prueba de comprensión lectora sobre la unidad didáctica, elaboración propia 2021. 
Después de analizados los datos se puede comprobar un $73 \%$ de las preguntas analizadas son correctas mientras un $27 \%$ de las mismas son incorrectas. La estrategia planteada, ejecutada para mejorar la compresión lectora en los estudiantes del grado tercero en la sede R Jagüi fue buena. Concluyendo se puede afirmar que la teoría de Aidan Chambers se hizo evidente mediante la aplicación de la unidad didáctica en esta investigación, reflejada en el manejo de conocimientos previos, la organización, el material didáctico, el acompañamiento permanente de la docente en todo el proceso.

\section{Conclusiones}

La investigación logró el propósito de crear una estrategia para mejorar la comprensión lectora en los estudiantes del grado tercero sede $\mathrm{R}$ Jagüi del Colegio Jun XXIII, con la incidencia de la teoría de Aidan Chambers, que reflejados en los resultados, corroborando la validez, aplicabilidad de esta teoría así: La unidad didáctica en general, permitió evidenciar los alcances de la propuesta en la apertura de una estrategia para mejorar la comprensión lectora, a partir de las categorías desarrolladas en cada una de las sesiones planteadas, dando cumplimiento a los objetivos de esta investigación.

Así mismo, con las actividades ejecutadas los estudiantes afirman comprender de una mejor forma los textos leídos, aumentó la capacidad de conversar con los demás sobre las lecturas realizadas. A la par con lo anterior, el material didáctico suministrado a través de la virtualidad es de vital importancia para la motivación e interés por el aprendizaje y la comprensión de textos.

Con el ánimo de que los resultados del proceso de investigación no se agoten y potenciar cada vez más la estructuración de competencias para mejorar la comprensión lectora en los estudiantes, es óptimo mencionar las siguientes recomendaciones: A las directivas del Colegio Juan XXIII del municipio de Macaravita Santander, fortalezcan proyectos transversales. A los docentes en cada una de las áreas del conocimiento, implementar prácticas cotidianas actividades didácticas con el hábito de leer y analizar.

Al indagar sobre la capacidad de comprensión lectora de los estudiantes del grado tercero se puede establecer que era escasa su compresión e interpretación los textos leídos, sin embargo, basados en la teoría de Aidan Chambers y la implementación de la unidad didáctica diseñada para este fin, logró mejorar su porte de comprender lo leído y conversar con fluidez sobre la misma lectura.

De la misma manera se pudo verificar que utilizar teorías correctas en ambiente de aprendizaje, hace que el estudiante este motivado y dispuesto a adquirir su aprendizaje, reflejando en los avances paulatinos de las actividades en cada sesión de trabajo, a través de la aplicación de medios tecnológicos, estrategias innovadoras, acompañados y orientados por un docente facilitador, permite al discente obtener excelentes resultados.

\section{Reconocimiento}

Agradecimientos a la Maestría en Educación de la Facultad de Educación de la Universidad de Pamplona.

\section{Referencias}

Arias, J. (2021). El campesinado en la educación rural: un debate emergente. Pedagogía y Saberes, (54). https://doi.org/10.17227/pys.num54-10555

Chambers, A. (2007). Dime: los niños, la lectura y la conversación. Fondo de Cultura económica.

Durango-Herazo, Z. (2015). ¿Por qué es importante la investigación cualitativa en la educación? Portal De Las Palabras, 1, 43-46. http://revistas.curnvirtual.edu.co/index.php/portaldel aspalabras/article/view/595

Escudero, C. \& Cortez, L. (2018). Técnicas y métodos cualitativos para la investigación científica. Utmach. $\mathrm{http} / / /$ repositorio.utmachala.edu.ec/bitstream/48000/ 14208/1/Cap.2-

Evoluci\%C3\%B3n\%20de\%20la\%20investigaci\%C3 $\%$ B3n $\% 20$ cient $\%$ C3\%ADfica.pdf

Herrera, J. (2010). La investigación cualitativa. Universidad de Guadalajara. http://biblioteca.udgvirtual.udg.mx/jspui/handle/123 $456789 / 1167$ 
Icfes. (2018). Publicación de resultados Saber $3^{\circ}, 5^{\circ}$ y $9^{\circ}$. Ministerio de Educación de Colombia http://www2.icfesinteractivo.gov.co/ReportesSaber3 59/consultaReporteEstablecimiento.jspx

Montero, I y León, O. (2002). Clasificación y descripción de las metodologías de la investigación en psicología. Internacional Journal of Clinical and Health Psychology.

https://www.researchgate.net/publication/26420207 _Clasificacion_y_descripcion_de_las_metodologias _de_investigacion_en_Psicologia

Moreno-Muños, C. \& Valverde-Caravaca, R. (2004). Glosas didácticas. Revista Electrónica Internacional, 11(11), 170 https://www.um.es/glosasdidacticas/doces/14Carmelo.pdf

Pimienta-Lastra, R. (2000). Encuestas probabilísticas vs. no probabilísticas. Política y Cultura, 13, 263-276. https://www.redalyc.org/pdf/267/26701313.pdf

Puerto, M. L. (2015). El libro álbum. Magisterio, 1(1). https://www.magisterio.com.co/articulo/el-libroalbum-como-estrategia-para-la-comprension-lectora

Reyes, Y. (2017). Una vida dedicada a la pedagogía de la lectura desde la primera infancia. Cultura y primera infancia, 1(1). https://maguared.gov.co/yolandareyes/

Salazar, M. C. (1992). La investigación Acción Participativa, inicio $y$ desarrollo. Popular. https://nanopdf.com/download/investigacion-accionparticipativa_pdf

Salazar, S \& Ponce, D. (1999). Hábitos de lectura. 1a. ed. Instituto del Libro y la lectura.

Sandín, M.P. (2010). Investigación Acción. S, Nieto. Principios, técnicas y métodos esenciales para la investigación cualitativa. Pp. $525 \quad-556$. http://media.picalab.cl/repo/descargas/bibliografia/ Metodolog\%C3\%ADa\%20de\%20Investigaci\%C3\% $\mathrm{B} 3 \mathrm{n} / \mathrm{Sand} \% \mathrm{C} 3 \% \mathrm{ADn}$.Investigaci $\% \mathrm{C} 3 \% \mathrm{~B} 3 \mathrm{n}$ Acci\%C3\%B3n.pdf

Silva de Marco, J. (2004). El texto conversacional. http://www.a43d.com.uy/jenny/el-texto-

Strauss, A., \& Corbin, J. (2002). Bases de la investigación cualitativa (Vol. 2). Universidad de Antioquia. 